\title{
CRITERIO DE TURBULENCIA Y ESTIMATIVAS DE LA ECUACIÓN BURGERS
}

JORGE HORNA MERCEDES, JONY DIONICIO VEREAU, RAÚL MARTINEZ ZOCÓN, AZUCENA ZAVALETA QUIPUSCOA

Resumen. A lo largo de la historia el estudio de turbulencia en dinámica de fluidos, no ha conseguido lograr una definición única o criterio teórico para este importante fenómeno. En este artículo, resolveremos en una y dos dimensiones la ecuación clásica de dinámica de fluidos, la ecuación de Burgers y aplicaremos los criterios de Turbulencia hecho por Ruelle [2], Muriel [3] y Getreuer, Albano y Muriel [6]. Además daremos algunas estimativas de la ecuación de Burgers.

Palabras Claves. Ecuación de Burgers, turbulencias, estimativas.

1. Introducción. Las ecuaciones diferenciales parciales, expresan muchas leyes fundamentales de la naturaleza. Uno de los principales impulsos para el desarrollo de las ecuaciones diferenciales parciales ha sido el estudio de problemas de propagación de ondas, estos problemas se originan en diversas áreas de la matemática aplicada, física e ingeniería, incluyendo dinámica de fluidos, óptica no lineal y mecánica de sólidos. En esa diversidad, las ecuaciones de Navier-Stokes, son las que modelan el movimiento de fluidos perfectos y viscosos incompresibles pero nuestra atención y punto de partida es el estudio de la ecuación de Burgers, que resulta ser la ecuación Navier-Stokes, sin gradientes de presión y volumen de fuerza.

La ecuación de Burgers:

$$
u_{t}+u u_{x}=v u_{x x}, \quad v>0
$$


fue presentada por J.M. Burgers como el modelo más simple para las ecuaciones diferenciales del flujo de fluido, posteriormente E. Hopf (1950) discutió las propiedades de la solución de la ecuación Burgers transformando la ecuación, en la ecuación de calor (transformación Cole - Hopf) [15].

La ecuación de Burgers aparece en la dinámica de gases y flujos de tráfico y que sirve como prototipo de ecuación de ondas no lineal para estudiar las llamadas Ondas de Choque que son discontinuidades que pueden aparecer en tiempo finito y después se propagan de manera regular. La ecuación de Burgers se utiliza a menudo como la ecuación más sencilla que exhibe los efectos no lineales de una distribución de energía y los efectos difusivos de la viscosidad sobre una región localizada para la propagación plena. Una propiedad útil de la ecuación de Burgers es que para ciertos valores del tiempo y viscosidad, la solución exacta puede ser generada numéricamente usando la transformada de Cole-Hopf y permite ver la eficacia de los métodos numéricos comparando con la solución exacta. Esto explica la gran cantidad de trabajos que se han hecho al respecto; la propagación de caos para la ecuación de Burgers ([16]), [20]), la dinámica en soluciones espectrales de ecuaciones de Burgers [2], la ecuación de Burgers, también está asociada a la dinámica poblacional [21].

Uno de los fenómenos mas interesantes en dinámica de fluídos es el de turbulencia. Hasta el momento no hay un único criterio teórico universalmente aceptable para la ocurrencia de turbulencia. Sin embargo Ruelle [2] conjeturó que cuando la derivada de la velocidad con respecto al tiempo tiende al infinito, el sistema se forma turbulento. Un criterio menos exigente fue puesto por Muriel [3], el cual la velocidad cambia de orientación, puede resultar turbuelencia, criterio que ha sido observado por experimentos $[4,5]$. El criterio mas reciente es hecho por Ge- 
treuer, Albano y Muriel [6], un campo de velocidad multivaluada puede representar turbulencia. En este trabajo analizamos cada una de las conjeturas usando como modelo la ecuación de Burgers. Además daremos algunas estimativas de la ecuación de Burgers.

\section{Material y Métodos}

\subsection{La Ecuación de Burgers. Criterios de Turbulencia}

La ecuación de Navier-Stokes puede ser escrita por:

$$
\rho\left(\frac{\partial v}{\partial t}+(v \cdot \nabla) v\right)=-\nabla p+\nabla \cdot T+f
$$

Donde $\rho$ representa densidad masa del fluido, $v$ es la velocidad, $p$ es la presión, $T$ es el tensor fuerza, y $f$ representa la suma de otras fuerzas.

Hacemos las siguientes simplificaciones

$$
\begin{gathered}
\nabla . T=\mu \nabla^{2} v \\
f=0 \\
\nabla \rho=0
\end{gathered}
$$

en otras palabras consideramos un fluido incompresible (2) sin fuerzas externas (3) ni gradiente de presión (4). Resultando la ecuación de Burgers

$$
\rho\left(\frac{\partial v}{\partial t}+(v \cdot \nabla) v\right)=\mu \nabla^{2} v
$$

En nuestro estudio primero consideramos el caso estacionario y unidimensional, es decir

$$
\rho\left(v \frac{\partial v}{\partial x}\right)=\mu \frac{\partial^{2} v}{\partial x^{2}}
$$

y su solución está dada por

$$
v(x)=\sqrt{\frac{2 C_{1} \mu}{\rho}} \tan \left(\sqrt{\frac{2 C_{1} \rho}{2 \mu}}\left(x+C_{2}\right)\right) .
$$


Imponiendo condición de frontera periódicas

$$
v(x)=v(x+L)
$$

obtenemos el valor de la constante $C_{1}$ :

$$
C_{1}=\frac{2 \mu \pi^{2} k^{2}}{\rho L^{2}}, \quad k \in \mathbb{Z}, \quad k \neq 0
$$

Además agregamos que $v(0)=0$, obtenemos

$$
v_{k}(x)=\frac{2 \mu k \pi}{\rho L} \tan \left(\frac{k \pi x}{L}\right)
$$

Se ha obtenido una familia de soluciones indexadas por el entero $k$. Esto significa que el campo de velocidad puede ser ahora ser considerado como una ecuación multi-valorada.

Ahora tenemos el caso anterior pero en dos dimensiones

$$
\begin{aligned}
& \rho\left(u_{x} \frac{\partial u_{x}}{\partial x}+u_{y} \frac{\partial u_{x}}{\partial y}\right)=\mu\left(\frac{\partial^{2} u_{x}}{\partial x^{2}}+\frac{\partial^{2} u_{x}}{\partial y^{2}}\right) \\
& \rho\left(u_{x} \frac{\partial u_{y}}{\partial x}+u_{y} \frac{\partial u_{y}}{\partial y}\right)=\mu\left(\frac{\partial^{2} u_{y}}{\partial x^{2}}+\frac{\partial^{2} u_{y}}{\partial y^{2}}\right)
\end{aligned}
$$

Donde la función velocidad está dada por $\mu=u_{x} i+u_{y} j=\left(u_{x}, u_{y}\right)$. Las soluciones para este sistema de ecuaciones diferenciales están dadas por:

$$
\begin{gathered}
u_{x}(x, y)=C_{4}+C_{5} \tanh \left(C_{1}+C_{2} x+C_{3} y\right) \\
u_{y}(x, y)=-\frac{C_{2} C_{4}}{C_{3}}-\frac{\rho C_{2} C_{5}+2 u\left(C_{2}^{2}+C_{3}^{2}\right)}{\rho C_{3}} \tanh \left(C_{1}+C_{2} x+C_{3} y\right)
\end{gathered}
$$

Las constantes arbitrarias $C_{1}, C_{2}$ y $C_{3}$ son imaginarias, (es decir, $C_{j}=i k_{j}$, donde $k_{j}$ es real) dejando una tangente hiperbólica para ser transformada en una función tangente:

$$
u_{x}(x, y)=C_{4}+C_{5} \tanh \left(k_{1}+k_{2} x+k_{3} y\right)
$$




$$
u_{y}(x, y)=-\frac{k_{2} C_{4}}{k_{3}}-\frac{\rho k_{2} C_{5}+2 u\left(k_{2}^{2}+k_{3}^{2}\right)}{\rho k_{3}} \tanh \left(k_{1}+k_{2} x+k_{3} y\right)
$$

Ahora podemos imponer la condición de frontera a lo largo de las direcciones $x, y$ similar al realizado en el caso unidimensional, obteniendo

$$
\begin{gathered}
u_{x}(x, y)=k_{4}+k_{5} \tan \left(k_{1}+\frac{k_{x} \pi}{L_{x}} x+\frac{k_{y} \pi}{L_{y}} y\right) \\
u_{y}(x, y)=\frac{L_{y}\left(\rho \frac{k_{x} k_{5}}{L_{x}}, 2 \mu \pi\left(\left(\frac{k_{x}}{L_{x}}\right)^{2}+\left(\frac{k_{y}}{L_{y}}\right)^{2}\right)\right)}{\rho k_{y}} \tan \left(k_{1}+\frac{k_{x} \pi}{L_{x}} x+\frac{k_{y} \pi}{L_{y}} y\right)-\frac{k_{x} L_{y} k_{4}}{L_{x} k_{y}}
\end{gathered}
$$

Estas soluciones 2-dimensionales son ahora indexadas por dos enteros, $k_{x} \mathrm{y}$ $k_{y}$, el cual puede ser considerada como multivaluada.

La ecuación de Burgers dimensión uno dependiente del tiempo toma la forma

$$
\rho\left(\frac{\partial v}{\partial t}+v \frac{\partial v}{\partial x}\right)=\mu \frac{\partial^{2} v}{\partial x^{2}}
$$

con la correspondiente solución

$$
v(x, t)=-\frac{C_{3}}{C_{1}}-\frac{2 \mu C_{2}}{\rho} \tanh \left(C_{1}+C_{2} x+C_{3} t\right)
$$

Observamos que con la elección de parámetros $C_{1}=0, C_{2}=\frac{i k \pi}{L}$ y $C_{3}=0$, la solución (20) se reduce al resultado obtenido previamente (10). Además, haciendo las constantes arbitrarias $C_{1}, C_{2}$ y $C_{3}$ imaginarias como anteriormente, obtenemos una expresión involucrando a la tangente en vez de la tangente hiperbólica

$$
v(x, t)=-\frac{k_{3}}{k_{2}}-\frac{2 \mu k_{2}}{\rho} \tan \left(k_{1}+k_{2} x+k_{3} t\right)
$$

La derivada con respecto al tiempo de la solución (21) es

$$
\frac{\partial v}{\partial t}=-\frac{2 \mu k_{2} k_{3}}{\rho}\left(1+\tan ^{2}\left(k_{1}+k_{2} x+k_{3} t\right)\right)
$$


el cual se aproxima a infinito para valores particulares de $x$ y $t$.

En dos dimensiones, las ecuaciones de Burgers dependientes del tiempo son

$$
\begin{aligned}
\rho\left(\frac{\partial u_{x}}{\partial t}+u_{x} \frac{\partial u_{x}}{\partial x}+u_{y} \frac{\partial u_{x}}{\partial y}\right) & =\mu\left(\frac{\partial^{2} u_{x}}{\partial x^{2}}+\frac{\partial^{2} u_{x}}{\partial y^{2}}\right) \\
\rho\left(\frac{\partial u_{y}}{\partial t}+u_{x} \frac{\partial u_{y}}{\partial x}+u_{y} \frac{\partial u_{y}}{\partial y}\right) & =\mu\left(\frac{\partial^{2} u_{y}}{\partial x^{2}}+\frac{\partial^{2} u_{y}}{\partial y^{2}}\right) .
\end{aligned}
$$

Con las correspondientes soluciones

$$
\begin{gathered}
u_{x}(x, y)=-\rho C_{3} C_{6}+2 \mu\left(C_{2}^{2}+C_{3}^{2}\right) \tanh \left(C_{1}+C_{2} x+C_{3} y+C_{4} t\right)-\frac{C_{4}+C_{3} C_{5}}{C_{2}} \\
u_{y}(x, y)=C_{5}+C_{6} \tanh \left(C_{1}+C_{2} x+C_{3} y+C_{4} t\right)
\end{gathered}
$$

Podemos elegir constantes imaginarias con el propósito de que la tangente hiperbólica transforme la función en la tangente. Las soluciones transformadas son

$$
\begin{gathered}
u_{x}(x, y)=-\frac{\rho k_{3} k_{6}+2 \mu\left(k_{2}^{2}+k_{3}^{2}\right)}{\rho k_{2}} \tan \left(k_{1}+k_{2} x+k_{3} y+k_{4} t\right)-\frac{k_{4}+k_{3} k k_{5}}{k_{2}}, \\
u_{y}(x, y)=k_{5}+k_{6} \tan \left(k_{1}+k_{2} x+k_{3} y+k_{4} t\right) .
\end{gathered}
$$

Las derivadas con respecto al tiempo de las soluciones transformadas son

$$
\begin{gathered}
\frac{\partial u_{x}}{\partial t}=-\frac{k_{4}\left(\rho k_{3} k_{6}+2 \mu\left(k_{2}^{2}+k_{3}^{2}\right)\right)}{\rho k_{2}}\left(1+\tan ^{2}\left(k_{1}+k_{2} x+k_{3} y+k_{4} t\right)\right), \\
\frac{\partial u_{y}}{\partial t}=k_{4} k_{6}\left(1+\tan ^{2}\left(1+\tan ^{2}\left(k_{1}+k_{2} x+k_{3} y+k_{4} t\right)\right)\right) .
\end{gathered}
$$

Para ciertos valores de $y, x$ y $t$ las derivadas (29) y (30) tiende al infinito. Que de acuerdo al criterio de Ruelle presenta turbulencia.

\subsection{Ecuación de Burgers: Existencia Local}

Consideremos la ecuación de Burgers para su discusión

$$
\begin{aligned}
& u_{t}=u u_{x}+\varepsilon u_{x x}, \quad \varepsilon=\text { constante, } \quad \varepsilon>0 \\
& u(x, 0)=f(x), \quad f \in C^{\infty}, \quad f(x) \equiv f(x+1)
\end{aligned}
$$


para ello consideremos, el esquema iterativo

$$
\begin{gathered}
u_{t}^{n+1}=u^{n} u_{x}^{n+1}+\varepsilon u_{x x}^{n+1}, \quad \varepsilon=\text { constante }, \quad \varepsilon>0 \\
u^{n+1}(x, 0)=f(x), \quad n=0,1,2,3, \ldots
\end{gathered}
$$

donde $u^{0}(x, t) \equiv f(x)$. La función $u^{n}$ puede ser mostrada que converge y su límite puede resolver (1.1).

\subsection{Unicidad}

Lema 1. El problema de Cauchy periódico (1.1) tiene a lo más una solución clásica.

\section{Prueba.}

Sean $u$ y $v$ soluciones. La diferencia $w=u-v$ satisface

$$
w_{t}=\frac{1}{2}(\alpha w)_{x}+\varepsilon w_{x x}, \quad \alpha=u+v, \quad w(x, 0)=0
$$

De

$$
\left\langle w,(\alpha w)_{x}\right\rangle=\left\langle w, \alpha_{x} w+\alpha w_{x}\right\rangle=\left\langle w, \alpha_{x} w\right\rangle-\left\langle(\alpha w)_{x}, w\right\rangle
$$

uno encuentra que

$$
\left\langle w,(\alpha w)_{x}\right\rangle=\frac{1}{2}\left\langle w, \alpha_{x} w\right\rangle
$$

y luego

$$
\begin{aligned}
\frac{1}{2} \frac{d}{d t}\langle w, w\rangle & =\left\langle w, w_{t}\right\rangle=\frac{1}{2}\left\langle w,(\alpha w)_{x}\right\rangle-\varepsilon\left\langle w_{x}, x_{x}\right\rangle \\
& =\frac{1}{4}\left\langle w, \alpha_{x} w\right\rangle-\varepsilon\left\langle w_{x}, w_{x}\right\rangle \\
& =\frac{1}{4}\left|\alpha_{x}\right|_{\infty}\langle w, w\rangle
\end{aligned}
$$

La condición inicial $w(x, 0)=0$ implica $w \equiv 0$.

\subsection{Algunas Estimaciones}

Sea $u$ una solución $C^{\infty}$ de (1.1) definida para $0 \leq t \leq T$. Observamos que

$$
\frac{1}{2} \frac{d}{d t}\langle u, u\rangle=\left\langle u, u u_{x}\right\rangle-\varepsilon\left\|u_{x}\right\|^{2} .
$$


Como

$$
\left\langle u, u u_{x}\right\rangle=\left\langle u^{2}, u_{x}\right\rangle=-2\left\langle u, u u_{x}\right\rangle
$$

tenemos $\frac{d}{d t}\langle u, u\rangle \leq 0$ y obtenemos

$$
\|u(., t)\| \leq\|u(., 0)\|=\|f\|, \quad 0 \leq t \leq T
$$

Para mostrar las estimaciones, usamos la notación $u_{j}=\partial^{j} u / \partial x^{j}$. Así

$$
u_{1 t}=\left(u u_{x}\right)_{1}+\varepsilon u_{1 x x},
$$

$\mathrm{y}$

$$
\begin{aligned}
\frac{1}{2} \frac{d}{d t}\left\langle u_{1}, u_{1}\right\rangle & =\left\langle u_{1},\left(u u_{x}\right)_{1}\right\rangle-\varepsilon\left\|u_{2}\right\|^{2} \\
& =\frac{1}{2}\left\langle u_{1}, u_{1}^{2}\right\rangle-\varepsilon\left\|u_{2}\right\|^{2} \\
& \leq \frac{1}{2}\left|u_{1}\right|_{\infty}\left\|u_{1}\right\|^{2}-\varepsilon\left\|u_{2}\right\|^{2} .
\end{aligned}
$$

De acuerdo a lo anterior

$$
\left|u_{1}\right|_{\infty}^{2} \leq\left\|u_{1}\right\|^{2}+2\left\|u_{1}\right\|\left\|u_{2}\right\|
$$

Además, la estimación

$$
\left\|u_{1}\right\| \leq \frac{1}{2 \pi}\left\|u_{2}\right\|
$$

se deduce de la expansión de Fourier. Por lo tanto tenemos

$$
\left|u_{1}\right|_{\infty} \leq\left\|u_{2}\right\|
$$

La función $u_{2}$ satisface

$$
u_{2 t}=\left(u u_{x}\right)_{2}+\varepsilon u_{2 x x},
$$

y por lo tanto

$$
\begin{aligned}
\frac{1}{2} \frac{d}{d t}\left\langle u_{2}, u_{2}\right\rangle & =\left\langle u_{2},\left(u u_{x}\right)_{2}\right\rangle-\varepsilon\left\|u_{3}\right\|^{2} \\
& \leq\left\langle u_{2}, u u_{2 x}\right\rangle+3\left\langle u_{2}, u_{x} u_{2}\right\rangle
\end{aligned}
$$


Desde que

$$
\begin{aligned}
\left\langle u_{2}, u u_{2 x}\right\rangle & =-\left\langle\left(u_{2} u\right)_{x}, u_{2}\right\rangle \\
& =-\left\langle u_{2 x} u, u_{2}\right\rangle-\left\langle u_{2} u_{x}, u_{2}\right\rangle,
\end{aligned}
$$

obtnemos

$$
\frac{d}{d t}\left\langle u_{2}, u_{2}\right\rangle \leq\left\langle u_{2}, u_{x} u_{2}\right\rangle \leq 5\left|u_{x}\right|_{\infty}\left\|u_{2}\right\|^{2} \leq 5\left\|u_{2}\right\|^{3}
$$

Usando las notaciones

$$
y(t)=\left\|u_{2}(., t)\right\|^{2}, \quad \phi(y)=5 y^{3 / 2}
$$

tenemos la desigualdad diferencial

$$
y^{\prime}(t) \leq \phi(y(t)), \quad 0 \leq t \leq T .
$$

Para estimar $y(t)$, necesitamos el siguiente lema.

Lema 2. Sea $\phi \in C^{1}[0, \infty\rangle$, con $y(t)$ y $y_{0}(t)$ que denotan funciones $C^{1}$ no negativas definida para $0 \leq t \leq T$. Si

$$
\begin{aligned}
& y^{\prime}(t) \leq \phi(y(t)), \quad y_{0}^{\prime}(t)=\phi\left(y_{0}(t)\right), \quad 0 \leq t \leq T \\
& y(0) \leq y_{0}(0),
\end{aligned}
$$

entonces $y(t) \leq y_{0}(t)$ en $0 \leq t \leq T$.

\section{Prueba.}

$$
\begin{aligned}
\phi(y)-\phi\left(y_{0}\right) & =\int_{y_{0}}^{y} \phi^{\prime}(v) d v \\
& =\left\{\int_{0}^{1} \phi^{\prime}\left(y_{0}+\left(y-y_{0}\right) s\right) d s\right\}\left(y-y_{0}\right) .
\end{aligned}
$$

Sea

$$
\eta(t)=y(t)-y_{0}(t), \quad C(t)=\int_{0}^{1} \phi^{\prime}\left(y_{0}(t)+\left(y(t)-y_{0}(t)\right) s\right) d s
$$




$$
\eta^{\prime}(t) \leq \phi(y(t))-\phi\left(y_{0}(t)\right)=C(t) \eta(t)
$$

Si consideramos

$$
z(t)=\exp \left(-\int_{0}^{t} e(\tau) d \tau\right) \eta(t),
$$

entonces $z^{\prime}(t) \leq 0$ y $z(0)=\eta(0) \leq 0$. Por lo tanto $z(t) \leq 0$, y además $\eta(t) \leq 0$. Para aplicar el lema, notamos que la solción $y_{0}(t)$ de

$$
y_{0}^{\prime}(t)=5 y_{0}^{3 / 2}, \quad y_{0}(0)=\left\|f_{x x}\right\|^{2}
$$

es creciente, y si $y_{0}(0)>0$, entonces existe un $T_{\infty}>0$ con

$$
\lim _{t \rightarrow T_{\infty}} y_{0}(t)=\infty
$$

El lema previo nos da un límite para $\left\|u_{2}(., t)\right\|$ es cualquier intervalo $0 \leq t \leq$ $T<T_{\infty} . \mathrm{A}\left\|u_{1}(., t)\right\| \mathrm{y}\|u(., t)\|$ son también limitados para $0 \leq t \leq T<T_{\infty}$. Podemos resumir mediante.

Lema 3. Considere (1.1) con $\varepsilon \geq 0$, existe un tiempo $T>0$ y una constante $K_{2}$, ambas dependiendo solamente sobre $\|f\|_{H^{2}}$, con la propiedad siguiente:

Si una solución $u(x, t)$ está definida para $0 \leq t \leq T$ entonces

$$
\|u(., t)\|_{H^{2}} \leq K_{2} \quad \text { en } \quad 0 \leq t \leq T .
$$

Lema 4. Supongamos que u es una solución $C^{\infty}$ de (1.1) definida para $0 \leq$ $t \leq T$, T es fijada como en el lema previo. Para $j=2,3, \ldots$ existe una constante $K_{j}$, dependiendo solamente sobre $\|f\|_{H^{j}}$, con

$$
\|u(., t)\|_{H^{j}} \leq K_{j} \quad \text { en } \quad 0 \leq t \leq T .
$$

\section{Prueba.}

Usaremos la prueba de inducción sobre $j$. El caso $j=2$ es tratado en el lema 
previo; para $j \geq 3$ tenemos:

$$
u_{j t}=\left(u u_{x}\right)_{j}+\varepsilon u_{j x x}
$$

implica que

$$
\frac{1}{2} \frac{d}{d t}\left\langle u_{j}, u_{j}\right\rangle=\left\langle u_{j},\left(u u_{x}\right)_{j}\right\rangle-\varepsilon\left\|u_{j+1}\right\|^{2} .
$$

Así

$$
\left(u u_{x}\right)_{j}=\sum_{\nu=0}^{j}\left(\begin{array}{c}
j \\
\nu
\end{array}\right) u_{\nu} u_{j+1-\nu} .
$$

Los términos $\left\langle u_{j}, u_{\nu} u_{j+1-\nu}\right\rangle$ puden ser estimados como sigue:

Para $\nu=1, \ldots, j-2$,

$$
\begin{aligned}
\left\langle u_{j}, u_{\nu} u_{j+1-\nu}\right\rangle & \leq\left|u_{\nu}\right|_{\infty}\|u\|_{H^{j}}^{2} \\
& \leq\|u\|_{H^{j-1}}\|u\|_{H^{j}}^{2} .
\end{aligned}
$$

$\operatorname{Para} \nu=j-1$ y $\nu=j$,

$$
\begin{aligned}
\left\langle u_{j}, u_{\nu} u_{j+1-\nu}\right\rangle & \leq\left|u_{j+1-\nu}\right|_{\infty}\|u\|_{H^{j}}\|u\|_{H^{\nu}} \\
& \leq\|u\|_{H^{j-1}}\|u\|_{H^{j}}^{2} \quad(j \geq 3) .
\end{aligned}
$$

Para $\nu=0$,

$$
\begin{aligned}
\left\langle u_{j}, u u_{j+1}\right\rangle & =-\left\langle\left(u_{j} u\right)_{x}, u_{j}\right\rangle \\
& =-\left\langle u_{j+1} u, u_{j}\right\rangle-\left\langle u_{j} u_{x}, u_{j}\right\rangle
\end{aligned}
$$

Por lo tanto

$$
\begin{aligned}
\left\langle u_{j}, u u_{j+1}\right\rangle & =-\frac{1}{2}\left\langle u_{j} u_{x}, u_{j}\right\rangle \\
& \leq\|u\|_{H^{j-1}}\|u\|_{H^{j}}^{2}
\end{aligned}
$$


Si $C_{j}$ es una constante numérica conveniente, encontramos que:

$$
\begin{aligned}
\frac{d}{d t}\left\|u_{j}\right\|^{2} & \leq C_{j}\|u\|_{H^{j-1}}\|u\|_{H^{j}}^{2} \\
& \leq C_{j}\|u\|_{H^{j-1}}\left(\left\|u_{j}\right\|^{2}+\|u\|_{H^{j-1}}^{2}\right) \\
& \leq C_{j} K_{j-1}\left(\left\|u_{j}\right\|^{2}+K_{j-1}^{2}\right)
\end{aligned}
$$

Puesto que tenemos limitado el espacio de todas las derivadas de $u$ en $0 \leq$ $t \leq T$, podemos usar la ecuación diferencial $u_{t}=u u_{x}+\varepsilon u_{x x}$ para limitar todas las derivadas con respecto a $t$ y las derivads mixtas.

\section{Resultados.}

- El problema de Cauchy 1-periodico para la ecuación de Burgers tiene solución única de clase $C^{\infty}$.

- La ecuación de Burgers satisface la conjetura de Ruelle.

- La conjetura de Albano y Muriel es verificada por la ecuación de Burgers.

- Presentamos estimativas para el espacio de derivadas de $u$.

\section{Conclusiones.}

- No existe un críterio teoríco universal que sea único para la ocurrencia de turbulencia.

- Las estimativas formuladas para la Ecuación de Burgers estan definidas localmente.

- Los críterios de turbulencia expuestos por Ruelle, Muriel y Getreuer, Albano y Muriel son verificados por la Ecuación de Burgers.

5. Agradecimiento. Los autores agradecen a todos los colegas y estudiantes que contribuyeron a preparar este trabajo. 


\section{Referencias Bibliográficas.}

[1] R. Aebi. Propagation of Chaos in entropy, Kodai Math. J. Volume 19, Number 3, 308-321, (1996).

[2] M. Basto, F. Calheiros. Dynamics in Spectral Solutions of Burgers Equation. Journal of Computational and Mathematics. Volume 205, Issue (August 2007).

[3] H. Brézis. Análisis Funcional Alianza Editorial S.A. Madrid, 1984.

[4] P. Calderoni, M. Pulvirenti. Propagation of Chaos for Burgers Equation, Annals of Institute Henri Poincaré, 39 No. 1 (1983), p. 85-97.

[5] P. Constantin, C. Foias, R. Teman. Attractors Representing Turbulent Flows, Memoirs AMS; 53, 1985.

[6] G. Da Prato, J. Zabczyk. Ergodicity for Infinite Dimensional Systems, London Mathematical Lecture Note Series (No 229) June, 1996.

[7] I. Davies, A. Truman, H. Zhao. Stochastic Heat and Burgers Equations and Their Singularities II - Analytical Properties and Limiting Distributions, University of Wales Swanres, UK, 2004.

[8] C. Foias, O. Manley, R. Temam. Approximate Inertial Manifolds and Effective Viscosity in Turbulent Flows, Phys. Fluids A 3(5), 1991, 898-911.

[9] J. Fritz. Formation of Singularities in One-Dimensional Nonlinear Wave Propagation, Communications on Pure and Applied Mathematics, Volume XXVII, 377405, 1974.

[10] E. Gutkin, M. Kac. Propagation of Chaos and the Burgers Equation, Siam Journal on Applied Mathematics (ISSN 0036-1399), vol. 43, Aug. 1983. p. 971-980.

[11] B. Goldys, B. Maslowski. Exponential Ergodicity For Stochastic Burgers and 2D Navier-Stokes Equations, Discovery Grant DP0346406, the UNSW Facul- 
[12] A. Gottlieb. Markov Transitions and the Propagation of Chaos, Computing Sciences Directorate Lawrence Berkeley National Laboratory. December 1998.

[13] J. Hale. Dynamics of a Scalar Parabolic Equation, Quarterly Volume 12 (3\&4) 1999, pp. 239-314.

[14] J. Hale, L. Magalhaes, W. Oliva. Dynamics in Infinite Dimensions, Applied Mathematical Sciences Volume 47 2002, Springer- Verlag. New York, Inc.

[15] E. Hopf. The Partial Differential Equation, Comm. Pure Appl. Math. 3, pp. 201-230.

[16] B. Jourdain. Propagation of Chaos and Poincaré Inequalities for a System of Particles Interacting Through Their Edf, The Annals of Applied Probability 2008, vol. 18, No 5, 1706-1736. Institute of Mathematical Statistics, 2008.

[17] T. Kato. Perturbation Theory of Linear Operators, Springer-Verlag, New York, 1966.

[18] H. Kreiss, J. Lorenz. Initial-Boundary Value Problems and the NavierStokes Equations, Siam 2004.

[19] X. Lui. On Approximate Inertial Manifolds for Two and Three Dimensional Turbulent Flows, J: Math. Annals. Appl. 63, 1992, p 559-580.

[20] R. Mikulevicias, B. Rozovskii. Stochastic Navier-Stokes-Equations, Propagation of Chaos and Statistical Moments, Center for Applied Mathematical Sciences 2001. 